\title{
Variación del componente graso y muscular mediante la aplicación de ejercicios funcionales en futbolistas profesionales
}

\section{Variation of the fat and muscle component through the application of functional exercises in professional soccer players}

\author{
Cristian Alejandro Naranjo-Sánchez \\ cristian.naranjo.18@est.ucacue.edu.ec \\ Universidad Católica de Cuenca, Azogues \\ Ecuador \\ https://orcid.org/0000-0001-7646-2664 \\ Raúl Fernando Moscoso-García \\ rfmoscosog@ucacue.edu.ec \\ Universidad Católica de Cuenca, Azogues \\ Ecuador \\ https://orcid.org/0000-0002-6113-8797 \\ Antonio Ricardo Rodríguez-Vargas \\ antonio.rodriguezv@ucacue.edu.ec \\ Universidad Católica de Cuenca, Azogues \\ Ecuador \\ https://orcid.org/0000-0002-4263-6686
}

Recepción: 30 de agosto 2021 Revisado: 20 de septiembre 2021 Aprobación: 15 de noviembre 2021 Publicación: 01 de diciembre 2021 
Revista Arbitrada Interdisciplinaria KOINONIA

Año VI. Vol VI. N4. Edición Especial: Educación III. 2021

Hecho el depósito de Ley: FA2016000010

ISSN: 2542-3088

FUNDACIÓN KOINONIA (F.K). Santa Ana de Coro. Venezuela.

Cristian Alejandro Naranjo-Sánchez; Raúl Fernando Moscoso-García; Antonio Ricardo Rodríguez-Vargas

\title{
RESUMEN
}

El presente estudio tuvo como fin analizar la variación del componente graso y muscular, mediante la aplicación de un programa de entrenamiento funcional, en futbolistas profesionales. De tipo descriptivo. Se aplicó (Test de valoración física, Vo2 Max, fuerza explosiva, fuerza resistencia, potencia, ejercicios preventivos, pliometría, resistencia aeróbica y anaeróbica, intervalos, intermitentes, ejercicios coordinativos y velocidad); por último, se realizó la toma de medidas del componente graso y muscular finales para la comparación pre y post entrenamiento funcional con el objetivo de conocer la variación de los mismos utilizando el programa estadístico SPSS versión 27. Se analizó la variación del componente graso y muscular, mediante la aplicación de un programa de entrenamiento funcional, en futbolistas profesionales, encontrando una variación significativa $(p<0,05)$ antes y después del trabajo realizado mediante los ejercicios funcionales en los componentes corporales de IMC, pliegues, Porcentaje Graso (Carter, a partir de Yuhasz) y Porcentaje Muscular (Poortmans).

Descriptores: Aprendizaje activo; método de aprendizaje; educación física. (palabras tomadas del Tesauro UNESCO).

\begin{abstract}
The purpose of this study was to analyze the variation of the fat and muscle component, through the application of a functional training program, in professional soccer players. Descriptive type. It was applied (Physical assessment test, Vo2 Max, explosive strength, resistance strength, power, preventive exercises, plyometrics, aerobic and anaerobic resistance, intervals, intermittent, coordination exercises and speed); Lastly, the measurements of the final fat and muscle component were taken for the comparison before and after functional training in order to know their variation using the statistical program SPSS version 27 . The variation of the fat and muscle component was analyzed. , through the application of a functional training program, in professional soccer players, finding a significant variation $(p<0.05)$ before and after the work performed through functional exercises in the body components of BMI, skinfolds, Fat Percentage (Carter, from Yuhasz) and Muscle Percentage (Poortmans).
\end{abstract}

Descriptors: Activity learning; learning methods; physical education. (Words taken from the UNESCO Thesaurus). 


\section{INTRODUCCIÓN}

El desarrollo óptimo y competitivo de los deportistas en relación con la variación del componente graso muscular es un elemento esencial dentro del rendimiento deportivo; por lo que, para que esta correlación sea armónica depende de la buena preparación que se debe dar en el proceso de entrenamiento y preparación física. El fútbol está presente en todo el mundo y forma parte de las competiciones olímpicas desde 1900. El juego se desarrolla lapsos de 45 minutos que se intercalan en 15 minutos intervalo de descanso entre ellos. Sin embargo, en un deporte de equipo como el fútbol, la exigencia de cambios frecuentes en el tipo de movimientos, por ejemplo, caminar, correr, esprintar, saltar, encarar, la velocidad (aceleraciones, desaceleraciones), dirección y tareas técnicas presenta un perfil de actividad que es intermitente por naturaleza. (Slimani \& Nikolaidis, 2019)

Para un equipo de fútbol, hay muchos factores importantes para el éxito y es difícil separar las características antropométricas y fisiológicas como factores cruciales en el rendimiento deportivo. Sin embargo, la evaluación de la composición corporal en los jugadores de fútbol ayuda a mejorar su rendimiento y a evaluar los resultados del plan de entrenamiento aplicado, que es un componente importante del proceso de entrenamiento individualizado y periodizado de los deportistas. Aunque existe una relación entre la composición corporal y la edad, no parece haber consenso si dicha asociación puede ser negativa o positiva. Es posible encontrar descensos del componente graso y un incremento en la más que se encuentra libre de grasa en relación con la edad, sin embargo, también se encuentra lo contrario en poblaciones similares. (Leão et al., 2019)

También existe una relación entre algunas características antropométricas, concretamente la masa grasa y el riesgo de lesiones. Además de la relación con el riesgo de lesiones, también es posible encontrar una relación entre la masa grasa y algunas características fisiológicas de rendimiento, como la velocidad y la potencia. En este 
sentido, se conoce que un mayor porcentaje de grasa corporal (Body Fat -\%BF) se asocia negativamente con la velocidad a más de 20 metros, una importante variable determinante en el rendimiento de los futbolistas. A lo largo de toda una temporada se puede encontrar diferencias en el \% BF pero no necesariamente en el peso. (Leão et al., 2019)

Además, ya se han encontrado divergencias en la grasa corporal entre las posiciones de los jugadores, lo que indica una especificidad posicional. Es importante tener en cuenta esta cuestión cuando se comparan los promedios de \%BF en los jugadores de fútbol. Por lo tanto, la evaluación de la composición corporal en los jugadores de fútbol de élite puede ayudar a mejorar el rendimiento y a seguir los resultados de los regímenes de entrenamiento aplicados. Sin embargo, la evaluación de la composición corporal incorpora algunas dificultades. (Leão et al. 2019)

Cada técnica presenta ventajas, sin embargo, también tiene limitaciones. Se conoce que existe una amplia gama de métodos utilizados, sin estandarización, que conducen a resultados diferentes, lo que hace que a menudo sea imposible hacer comparaciones entre varios estudios. Las medidas antropométricas se utilizan globalmente en la monitorización del entrenamiento como un importante determinante del rendimiento. Sin embargo, la literatura es escasa en lo que respecta a los datos epidemiológicos en este contexto específico y es necesario un debate metodológico sobre los procedimientos de estimación para aclarar la aplicación de la práctica.(Leão et al. 2019)

Es bien sabido que la comprensión de los requisitos específicos del juego de fútbol de élite puede proporcionar información reveladora sobre lo que realmente se necesita para el éxito competitivo en ese deporte. En particular, las medidas antropométricas de la composición corporal, y las capacidades fisiológicas y físicas, incluyendo la resistencia muscular y respiratoria, además de la fuerza muscular y la flexibilidad mediante pruebas a los jugadores de fútbol. Estas medidas pueden complementarse entre sí, y su combinación puede proporcionar a los entrenadores de fútbol, de fuerza y de atletismo 
un mejor conocimiento de las características requeridas para participar con éxito en el nivel de élite. (Slimani \& Nikolaidis, 2019)

El mejor rendimiento en el deportista no solo depende de la preparación y la condición física, ya sea esta cualitativa como cuantitativamente, sino que también será el mismo en deportistas con condiciones físicas favorables, reflejado en el desarrollo de actividad el deporte. En relación con esto todos estos elementos cobran relevancia, debido a que cada carrera deportiva tiene un conjunto de requisitos que mayormente los deportistas deben tener, respecto a una determinada anatomía para lograr un deporte óptimo. (Rodríguez, et al. 2014)

Actualmente, el método Heath Carter es considerado el método de somatotipo de mayor uso, creado en 1964, mismo que se basa en las medidas cinéticas para obtener el somatotipo variando el método Sheldon; y ha demostrado que el modelo biológico no tiene dependencia con respecto a los aspectos genéticos sino que interviene otros factores extrínsecos entre ellos la nutrición y las actividades físicas, los cuales pueden ser modificados para un mejorar el rendimiento del deporte en cuestión.(Rodríguez, et al. 2014)

Según Rienzi y Mazza (1998) en el caso de los deportes como el fútbol sala y el fútbol, la posición de juego debe ser considerada fundamentalmente, tanto en términos de sus necesidades técnico-tácticas como fisiológicas en la morfología general. (Toala \& Aguilar, 2018)

Según Carter (1990) la determinación de los elementos de composición morfológica actual del deportista puede llevar a entender de mejor manera la composición y su relación con la actividad o rendimiento en eventos o competiciones. En estudios previos se ha observado cómo la morfología de los deportistas presenta un perfil antropométrico con diferencias en otras variables, principalmente en cuanto a altura, masa corporal y pliegues, y circunferencias, tanto para el deporte como para la posición de juego y se han observado notoriamente estas diferencias físicas. (Rivera, 2006) 
Según AlGindan, et al. (2014) la determinación de la composición corporal en la actividad deportiva es una técnica de fácil acceso, económica y de aplicación simple en varias disciplinas, la misma puede generar información relacionada con el tamaño corporal; así las medidas de componente de masa muscular son interesantes en razón de los cambios de las actividades físicas e influye en la composición corporal. De la Rosa, et al. (2010) mencionan que para cuantificar la masa, el músculo como la grasa, es necesario la cuantificación del peso lastre o extra en relación con el rendimiento mecánico, esto brindaría una mejor interpretación de los costos mecánicos y su efecto anabólico dentro del entrenamiento físico, además de la capacidad física, producción de fuerza y el rendimiento muscular.(Cárdenas-Fernández, 2015)

Como se ha observado la evaluación de la composición corporal busca determinar el porcentaje de masa magra (músculo) y masa grasa en el deportista como indicadores que pueden mejorar el rendimiento. Estos factores permiten valorar y conocer desarrollo evolutivo desde el aspecto físico del futbolista desde edades tempranas, aportando de manera significativa en los mismos, otorgando cambios morfológicos acordes con las características específicas y necesarias inmersas en el futbol profesional, considerando como factor principal los procesos de entrenamiento dirigidos y especializados (funcional) a la necesidad del deporte y al desarrollo físico adecuado del deportista para su óptimo rendimiento competitivo. Por lo que el fin de este estudio es analizar la variación del componente graso y muscular, mediante la generación de un entrenamiento funcional aplicado en un programa de actividades en futbolistas profesionales, cuya variación puede ser significativa respecto al entrenamiento propuesto. 


\section{Referencial teórico}

\section{Composición corporal}

En cuanto a la estimación de la composición corporal, el Instituto Cubano de Medicina del Deporte (IMD) se ha apoyado tradicionalmente en dos elementos fundamentales: la acumulación de grasa, que es la energía almacenada por el cuerpo, y la masa corporal activa (LCR) formada por tejidos con alta actividad metabólica, y que está relacionada con el consumo de oxígeno y la capacidad del sujeto para funcionar. Por ello, la actividad física o la ausencia de la altera más o menos dos de los componentes del cuerpo humano: masa grasa y masa muscular, por lo que está comprobado el uso por la agencia de evaluación del componente en el control médico del entrenamiento deportivo ha sido reconocido durante varias décadas. (Acosta \& García, 2013)

La masa grasa sirve como reserva de energía para el cuerpo, pero su concentración por encima y durante la fase de competición puede convertirse en una barrera frente al rendimiento de los atletas en los deportes especialmente en aquellos que tienen que estar físicamente activos (1921) a una amplia gama de parámetros fisiológicos como el volumen sanguíneo, la fuerza muscular, la viabilidad, la actividad física, el estrés oxidativo en reposo y durante la actividad física. (Acosta \& García, 2013)

Como parte del análisis del componente corporal y además de la valoración del desarrollo físico, se considera la medición de áreas musculares y grasas de diferentes partes del cuerpo, en particular se relaciona con la biomecánica con la ejecución de movimientos. Durante las últimas dos décadas, los avances tecnológicos han permitido el uso de herramientas muy precisas, así como el desarrollo de modelos matemáticos basados en: edad, peso, estatura, diámetro de huesos, circunferencia muscular y cutánea (parte de ecuaciones gráficas antropométricas y estadísticas) se utiliza para diagnosticar, controlar y corregir la composición corporal con fines deportivos. (Acosta \& García, 2013) 


\section{La cineantropometría}

Según Norton (1996) dentro de la antropometría la herramienta del mismo nombre que permite medir pliegues, peso, talla, diámetros, longitud y circunferencia que se usan para la medición del componente corporal (CC); los que son aplicables en relación con el protocolo de actuación y estimadores de la ecuación DC. Estos son conocidos como los métodos duales indirectos, así como mayormente las técnicas de evaluación de la composición corporal de la práctica deportiva. (Martínez \& Urdampilleta, 2012)

El órgano rector de referencia antropométrica recomienda que estas se basen a las mediciones de parámetros corporales, considerando a los concesos internacionales, (International Society for the Advancement of Kinantropometry) (ISAK, 2001), siguiendo enlaces específicos basados en las publicaciones de Ross y Marfell Jones en 1991, referidos en ISAK y por el Grupo Español de Cineantropometría (GREC). (Martínez \& Urdampilleta, 2012)

Según Manso (2003) la composición corporal inadecuada de un deportista puede impedirle alcanzar un rendimiento atlético máximo. (Martínez \& Urdampilleta, 2012) por otro lado, Martínez (2011), indica que los estudios antropométricos en el deporte permiten evaluar las características morfológicas (fitness, proporciones, CC, somatotipo) en la temporada de actividad física (Fases de preparación general, específicas y competición), con el objetivo de controlar por los aspectos antropométricos que pueden llegar a limitar el rendimiento deportivo y en el marco del seguimiento dietético y nutricional. Por lo tanto, la evaluación antropométrica proporciona aspectos útiles y prácticos para la experiencia en ciencias de la nutrición y el ejercicio, como una herramienta de consulta para evaluar y monitorear la higiene y el somatotipo corporal en el deporte.(Martínez \& Urdampilleta, 2012)

El examen se realizará en una habitación suficientemente grande y a una temperatura agradable. Los sujetos para estudiar estarán descalzos con ropa adecuada, como pantalones cortos o bikinis. Las medidas de altura y peso cambian durante el día, por lo 
que debe tomarlas primero por la mañana. Si esto no es posible, debe incluir la hora del día y las condiciones actuales, como la ingesta de alimentos o el entrenamiento previo. Para permitir la comparación de medidas en cualquier población, se tomarán en la mitad derecha del cuerpo. No obstante, si existe una limitación o preponderancia física en el crecimiento de alguna de las extremidades, se considerarán cuerpos indeformables. El material se calibrará y comprobará su precisión antes de comenzar las mediciones. La exploración comenzará marcando los puntos anatómicos y antropométricos requeridos para el estudio. Las mediciones se realizarán por orden de practicidad y conveniencia. Por ejemplo, las personas que marcan hojas antropométricas. (Martínez \& Urdampilleta, 2012)

Las mediciones deben repetirse al menos dos veces y una tercera si es necesario. En el primer caso se utiliza el promedio y en el segundo el promedio. Luego pídale ayuda al marcador. Informe ejercicio destinadas a mejorar el rendimiento deportivo.(Martínez \& Urdampilleta, 2012)

\section{Entrenamiento Funcional}

Según Gonzáles (2014) desde sus inicios, el ejercicio ha sido parte de la naturaleza humana. Sin duda, es en la antigua Grecia donde la actividad física jugó un papel fundamental en la búsqueda del desarrollo integral de la ciudadanía. Este ejercicio físico se organiza sistemáticamente como entrenamiento por primera vez y así lleva a los deportistas a mejorar su rendimiento deportivo. Hoy se menciona que el entrenamiento funcional es un proceso orientado al ejercicio y parte del proceso de rehabilitación, cuyo objetivo es incrementar las posibilidades de las personas para actuar en el entorno físico que las rodea.(Antunes et al., 2020)

Según Papí (2007) el entrenamiento funcional está destinado a mejorar las habilidades de un atleta en términos de tanto como sea posible en la fuerza, resistencia y equilibrio; y sus principales objetivos se reducen a incrementar el rendimiento físico y prevenir 
lesiones deportivas. El entrenamiento funcional es un entrenamiento con un objetivo específico, es decir, está diseñado y dirigido a conseguir un objetivo a través de una serie de ejercicios para mejorar la fuerza y las habilidades cognitivas de quienes lo utilizan.(Antunes et al., 2020)

\section{Ejercicios funcionales}

El entrenamiento de las cualidades físicas del futbolista siempre ha sido parte importante del contenido del entrenamiento futbolístico, ya sea dentro de un ciclo de entrenamiento; microciclo, mesociclo o macrociclo. A lo largo de la historia evolutiva del entrenamiento de las cualidades físicas, se han entrenado utilizando varios métodos respaldados por el conocimiento que ha adquirido la fisiología del movimiento (que es la base científica para el entrenamiento de las cualidades físicas, tanto primarias como secundarias). (Rivas et al., 2013) Por lo tanto, el entrenamiento funcional para jugadores de fútbol se centra en el desarrollo de la parte inferior del cuerpo (piernas y glúteos) y la parte superior del cuerpo (más énfasis en el abdomen). Este enfoque se deriva de que son los músculos que más se utilizan a la hora de practicar este deporte y que se pretenden fortalecer en el proceso de ejercicio brindando como resultado un mejor rendimiento evitando en la medida de las posibilidades posibles lesiones producto de la exigencia del deporte y sus movimientos. A continuación, se detallan algunos de los ejercicios que se aplican en la práctica de este deporte.

\section{Fartlek}

Consiste en correr para cambiar el tiempo o la velocidad. Se hace mejor en terrenos variados y terrenos abiertos, la frecuencia cardíaca aumentará y disminuirá. causado por altibajos. La duración puede variar considerablemente (hasta 40 minutos para los jugadores) y al mismo tiempo, los intentos realizados deben maximizarse por debajo del máximo. La oscilación del HR varía de 140 a 175 I / min. El rango de baja intensidad 
debe ser mayor o igual al decimal de intensidad y nunca menor, ya que esto hará que la recuperación del sea insuficiente para que la frecuencia cardíaca no disminuya.

\section{Aeróbico largo:}

Entrena hasta 15 minutos. La frecuencia cardíaca estará entre ,130 y 150 I/ min. Se pueden realizar hasta 4 repeticiones de 10 a 15 minutos. el tiempo de descanso vendrá determinado por el tiempo de trabajo. Su uso está dirigido principalmente a mejorar la resistencia en la zona subaeróbica.

\section{Aeróbicos cortos:}

Se utiliza un tiempo de trabajo de 3 a 5 minutos. La frecuencia cardíaca estará entre ,160 y $175 \mathrm{I} / \mathrm{min}$. Puedes hacer hasta 7 repeticiones en 5 minutos con pausas que van desde 90 a 180 segundos. para lograr la aclimatación en la zona hiperaeróbica.

\section{Intervalo:}

Este método utiliza una distancia relativamente corta. La humedad relativa será de,175 I / min aproximadamente. La pausa suele ser incompleta. Se recomienda su uso durante la fase preparatoria después del trabajo básico y durante el mantenimiento.

\section{Método Intermitente:}

La frecuencia cardíaca aumenta mientras se corre y no es estable durante los períodos de baja intensidad o de descanso, lo que resulta en un aumento constante, lo que resulta en una mejora de VO2 máx. (Motor aeróbico), permitirá al jugador de un partido dedicar más tiempo al metabolismo rítmico de la energía, evitando así superar el umbral de y producir demasiado ácido láctico. (Argemi, 2001) 


\section{Test de Cooper (basado en García M., Navarro M. y Ruiz J., 1998)}

Esta prueba dura 12 minutos y debe cubrirse en la medida de lo posible. Dependiendo de los resultados de esta prueba, es posible tener el VO2 máx. De forma indirecta. La fórmula para determinar el consumo máximo de oxígeno es: VO2 máx. $=22,351 \times \mathrm{D}$ en km 11,288.

\section{Entrenamiento de Hipertrofia:}

Este método de entrenamiento es la forma básica de entrenamiento de fuerza para todos los deportes. El objetivo principal de esto es el crecimiento de las fibras musculares (hipertrofia). La intensidad de la carga viene indicada por el porcentaje en peso con el que se procesa, que está entre 60 y 75\% R.M.

\section{Fuerza explosiva:}

Entrenamiento de resistencia: el entrenamiento de fuerza explosivo se puede realizar utilizando cargas útiles o pesos adicionales. Para estos, intensidades entre 30 y 70 R.M. Haga ejercicio a la máxima velocidad de ejecución.

Entrenamiento pliométrico: este método incluye el uso de movimientos de salto, salto, rebote y rebote, que estimulan la rápida contracción y expansión de las fibras musculares.

Entrenamiento de tracción: este método utiliza el tirón de un peso que es resistente a la carrera.

\section{Método Interválico:}

Para los futbolistas, se recomienda el método de intervalo corto. Se utiliza para cargas de trabajo con una duración de entre 15 y 60 segundos.

El entrenamiento por intervalos funciona de dos maneras para lograr cambios cardíacos durante la fase de ejercicio, la presión cardíaca alta provoca hipertrofia miocárdica, mientras que durante la fase de recuperación la actividad actúa principalmente a nivel del volumen cardíaco provocando la dilatación de las cavidades cardíacas. 
Además, el entrenamiento intensivo en intervalos, con una intensidad máxima de absorción de oxígeno de 90, y con una intensidad isotrópica máxima de 30, también ha llevado a una demanda selectiva de fibras FT.

\section{MÉTODO}

El diseño del presente estudio corresponde a un enfoque de investigación cuantitativo de tipo descriptivo, de corte longitudinal (Erazo, 2021). Para el análisis comparativo investigación sobre la variación del componente graso y muscular la población de estudio fue finita, con una muestra intencional no probabilística conformada por 20 futbolistas del Club Deportivo Profesional Oriental del cantón de Shushufindi, provincia de Sucumbíos. Para la medición antropométrica se utilizó el método de la International Society for the Advancement of Kineatropometry (ISAK) y de evaluación descritos por Norton \& Olds (1996) y Drinkwater (1984), el mismo que se aplicó en un pre y post entrenamiento de tipo funcional con una duración de 12 semanas aproximadamente.

Se utilizó el Kit para la aplicación del método antropométrico que consta de instrumentos y calibres: Campbell 10 (caliper corta), medidor de segmento retráctil, escuadra metálica, calibrador de plástico, cinta métrica, estadiómetro portátil, cajón antropométrico y una escala digital para peso con precisión de $100 \mathrm{~g}$. La recopilación de datos es facilitada por un asistente, que ayuda al evaluador a registrar las variables. El mismo está capacitado en técnicas de medición, lo que permitió el desarrollo de un trabajo fluido y rápido, acorde al número de sujetos y en el poco tiempo disponible, además de garantizar la correcta secuencia de los sitios de medición.

Se utilizó una hoja electrónica de recolección de datos, la cual fue diseñada con la intención de mejorar de brindar agilidad a la medición, con un orden que va de arriba hacia abajo de la posición anatómica. Las medidas corresponden a variables básicas, peso, altura de pie, altura sentada y envergadura de brazos, 3 diámetros óseos, 6 perímetros o contornos axiales y apendiculares y 8 pliegues cutáneos. La forma corporal 
de los sujetos se determinó mediante el método Carter Somatotype (Carter y Heath, 1990; Carter, 2002), las puntuaciones $Z$ del Phantom mediante las fórmulas de Ross (Ross y Wilson, 197) y la masa grasa mediante las fórmulas de Kerr. (Kerr, 1988).

Procedimiento para la medición del componente graso y muscular y la aplicación del entrenamiento funcional. A continuación, se explican las fases de la medición inicial de las variables dependientes, la aplicación del programa de entrenamiento funcional y la medición final de las variables dependientes. La toma de datos inicial se realizó los primeros días antes de iniciar la temporada competitiva.

Consecutivamente, se cumplieron aproximadamente 3 meses de entrenamiento, con una preparación habitual entre 2 y 3 sesiones a la semana con actividades coordinadas por el preparador físico (Test de valoración física, Vo2 Max, fuerza explosiva, fuerza resistencia, potencia, ejercicios preventivos, pliometría, resistencia aeróbica y anaeróbica, intervalos, intermitentes, ejercicios coordinativos y velocidad); por último se realizó la toma de medidas del componente graso y muscular finales para la comparación pre y post entrenamiento funcional con el objetivo de conocer la variación de los mismos con el uso del programa estadístico SPSS versión 27 (Erazo y Narváez, 2020).

\section{RESULTADOS}

El grupo de futbolistas del estudio estuvo conformado por deportistas del somatipo mesomórfico, con valores promedio de $60,68 \pm 7,54$ de masa corporal antes de empezar el entrenamiento con ejercicios funcionales, y con una talla promedio de $170,02 \pm 5,3 \mathrm{~cm}$; se destaca que el grupo estuvo conformado, además por un $60 \%$ de deportistas caucásicos o mestizos y un $40 \%$ de descendencia afro ecuatoriana. Se determinó en la comparación de pliegues (Tabla 1), diferencias estadísticamente significativas una vez establecido los ejercicios funcionales en los deportistas, destacando entre ellos cambios en los bíceps, cresta ilíaca, abdominales y muslo; es importante enfatizar además, que 
los valores de variación de los pliegues mostraron cambios porcentuales importantes como una tendencia importante de disminución, lo que daría a entender que el grupo se mostró mucho más consistente en las diferentes medidas con valores muy próximos entre ellos.

Tabla 1.

Comparación de pliegues pre y post entrenamiento funcional (3 meses).

\begin{tabular}{|c|c|c|c|c|c|c|c|c|c|}
\hline \multirow[b]{3}{*}{ Pliegues } & \multicolumn{4}{|c|}{ Pre - entrenamiento } & \multicolumn{4}{|c|}{ Post - entrenamiento } & \multirow[b]{3}{*}{ p } \\
\hline & \multirow[b]{2}{*}{ Media } & \multicolumn{2}{|c|}{ Median } & \multirow[b]{2}{*}{ CV } & \multirow{2}{*}{$\begin{array}{l}\text { Medi } \\
\text { a }\end{array}$} & \multicolumn{3}{|l|}{ Media } & \\
\hline & & $\mathbf{a}$ & DE & & & na & $\mathrm{DE}$ & CV & \\
\hline Triceps & 171,95 & 8,00 & 3,10 & $28 \%$ & 8,25 & 7,50 & 2,07 & $25 \%$ & 0,500 \\
\hline \multicolumn{10}{|l|}{ Subescap } \\
\hline \multirow[t]{2}{*}{ ular } & 10,25 & 9,00 & 3,93 & $38 \%$ & 9,4 & 9 & 2,56 & $27 \%$ & 0,133 \\
\hline & & & & & & & & & 0,011 \\
\hline Biceps & 4,70 & 4,00 & 0,92 & $20 \%$ & 5,1 & 5 & 0,96 & $19 \%$ & * \\
\hline Cresta & & & & & & & & & 0,012 \\
\hline ilíaca & 14,45 & 12,00 & 8,85 & $61 \%$ & 12,05 & 10 & 5,37 & $45 \%$ & * \\
\hline \multicolumn{10}{|l|}{ Supraespi } \\
\hline nal & 9,60 & 7,00 & 5,99 & $62 \%$ & 9,05 & 8,5 & 4,11 & $45 \%$ & 0,395 \\
\hline Abdomina & & & & & & & & & 0,018 \\
\hline \multirow[t]{2}{*}{ I } & 14,55 & 11,00 & 7,78 & $53 \%$ & 12,2 & 11 & 4,37 & $36 \%$ & * \\
\hline & & & & & & & & & 0,001 \\
\hline Muslo & 12,10 & 12,00 & 3,85 & $32 \%$ & 10,2 & 10 & 2,44 & $24 \%$ & * \\
\hline Pierna & 7,25 & 7,00 & 2,46 & $34 \%$ & 7,1 & 7 & 1,8 & $25 \%$ & 0,589 \\
\hline
\end{tabular}

* Significativamente diferente

$(p<0,05)$ 
Respecto a la comparación de perímetros (Tabla 2) se notaron como en el caso anterior disminución en los valores porcentuales del coeficiente de variación, y diferencias estadísticamente significativas después del ejercicio funcional en brazo flexionado y contraído, cintura, muslo medio, muslo corregido, pierna y pierna corregida; lo que en general mostraría un trabajo en las extremidades inferiores de los futbolistas, considerando que el mismo es de vital importancia para esta actividad qué tiene explosividad y cambios abruptos en el desarrollo de la actividad física.

Tabla 2.

Comparación de perímetros pre y post entrenamiento funcional (3 meses).

\begin{tabular}{|c|c|c|c|c|c|c|c|c|c|}
\hline & Pre - e & ntrenamie & nto & & Post - & entrenam & ento & & \\
\hline & Medi & & $\mathbf{D}$ & C & Medi & Median & $\mathbf{D}$ & C & \\
\hline Perímetros & $\mathbf{a}$ & Mediana & $\mathbf{E}$ & v & $\mathbf{a}$ & $\mathbf{a}$ & $\mathbf{E}$ & V & $\mathbf{p}$ \\
\hline & & & 2 & 11 & & & 1, & 6 & 0,19 \\
\hline Brazo relajado & 25,81 & 25,65 & 81 & $\%$ & 26,58 & 26,3 & 53 & $\%$ & 1 \\
\hline & & & 2 & 12 & & & 1, & 5 & 0,07 \\
\hline Brazo corregido & 22,95 & 23,6 & 65 & $\%$ & 23,99 & 23,93 & 29 & $\%$ & 9 \\
\hline Brazo flexionado y & & & 2 & 10 & & & 1 & 5 & 0,03 \\
\hline contraído & 27,58 & 28 & 82 & $\%$ & 28,34 & 28,55 & 55 & $\%$ & $7^{*}$ \\
\hline & & & 5 & 7 & & & 3 & 5 & 0,00 \\
\hline Cintura & 72,27 & 70,25 & 11 & $\%$ & 70,3 & 69,9 & 6 & $\%$ & $8^{*}$ \\
\hline & & & 4 & 5 & & & 6 & 7 & 0,77 \\
\hline Caderas & 88,48 & 86,75 & 82 & $\%$ & 87,17 & 87,6 & 16 & $\%$ & 8 \\
\hline & & & 4 & 8 & & & 3 & 7 & 0,00 \\
\hline Muslo medio & 49,81 & 49,35 & 11 & $\%$ & 51,19 & 51,4 & 8 & $\%$ & $3^{*}$ \\
\hline & & & 3 & 8 & & & 3 & 8 & 0,00 \\
\hline Muslo corregido & 46,01 & 46,92 & 74 & $\%$ & 47,99 & 48,55 & 61 & $\%$ & $1^{*}$ \\
\hline
\end{tabular}




\begin{tabular}{llllllllll} 
& & & 1, & 6 & & & 1, & 5 & 0,01 \\
Pierna & 34,08 & 34,15 & 9 & $\%$ & 34,64 & 34,55 & 75 & $\%$ & $4^{*}$ \\
& & & 1, & 6 & & & 1, & 6 & 0,01 \\
Pierna corregida & 31,8 & 31,8 & 91 & $\%$ & 32,41 & 32,26 & 85 & $\%$ & $1^{*}$ \\
\hline
\end{tabular}

* Significativamente diferente $(p<0,05)$

En lo que se refiere a los componentes corporales que mostrarían cambios significativos en los jugadores, se observa (Tabla 3), cambios en el índice de masa corporal, el porcentaje muscular, pliegues; en lo referente al porcentaje de grasa, el porcentaje muscular de los diferentes indicadores variaron en el índice de significancia siendo para el componente graso de Carter, el que mostró diferencias estadísticamente significativas este último se destaca como el de mayor relevancia dentro de los indicadores para medir el componente graso.

Tabla 3.

Valores de significancia componentes corporales entrenamiento funcional (3 meses)

\section{Significanci}

Componente corporal

IMC (Kg/m2)

Porcentaje Muscular (Poortmans)

Sumatorio de 6 pliegues

Sumatorio de 8 pliegues

Porcentaje Graso (Slaughter)

Porcentaje Graso (Faulkner, a partir de

Yuhasz)

Porcentaje Graso (Carter, a partir de

Yuhasz) a

$0,046^{*}$
$0,004^{\star}$
$0,021^{*}$
$0,024^{\star}$
0,055
0,058
$0,021^{*}$


* Significativamente diferente $(p<0,05)$

\section{DISCUSIÓN}

En el estudio de Miranda (2013) se evaluaron los efectos del programa de entrenamiento de fútbol de 10 semanas en, habilidades técnicas, psicológicas antropométricas y parámetros específicos de rendimiento en jugadores de fútbol juvenil, y se determinó que el entrenamiento generado mostró características similares de volumen e intensidad en ellos conduciendo a cambios significativos $(P \leq 0.05)$ en las pruebas de masa corporal, índice de masa corporal, masa corporal magra, entre otras que fueron medidas; en conclusión, con base en la importancia de las pruebas de técnica de fútbol para la identificación de talentos, los entrenadores de fútbol deben utilizar este tipo de evaluación durante la rutina de entrenamiento. Estos resultados se establecieron en un marco similar de temporalidad como el tomado en esta investigación además los valores de cambio en los índices de masa se mostraron similares respecto a la significancia estadística calculada tanto en índice de masa muscular como en otros indicadores, finalmente la conclusión es concordante respecto al criterio de una evaluación de la rutina al momento de entrenar y en la forma en que se equipararon las condiciones del grupo en general. (Miranda et al., 2013)

Hammami (2013) en su estudio de los efectos de una temporada de fútbol sobre las características antropométricas y la aptitud física en jóvenes futbolistas de élite encontró que la temporada de entrenamiento de fútbol pudo proporcionar una mejora libre de maduración en las características antropométricas y de rendimiento en jugadores de fútbol jóvenes durante la temporada de entrenamiento. En un estudio similar de Fessi (2016) en el que se analizó los cambios físicos y antropométricos durante la pretemporada y la temporada en futbolistas profesionales, aportando más pruebas sobre 
la eficacia del período de entrenamiento de pretemporada para alcanzar los picos de exigencia de la condición física de la temporada competitiva. Sin embargo, los entrenadores principales y los entrenadores de fuerza y acondicionamiento tienen que gestionar y controlar eficazmente la carga de entrenamiento para evitar superar los límites fisiológicos de los jugadores.

El resultado de dicho estudio confirma que las diferentes modalidades de entrenamiento, que incluyen la capacidad de resistencia, el entrenamiento intermitente con ejercicios genéricos y específicos y las magnitudes de entrenamiento pliométrico, pueden conducir a mejorar la aptitud física. Estos resultados se muestran relacionados a este estudio puesto que se notó una mejora significativa en los aspectos de equiparación de masa muscular y componente graso en los jugadores que se entrenaron de forma funcional, lo corroboraría el criterio de Fessi y Hammami.

Castrillón, et al. (2009) determinaron los resultados de un programa de entrenamiento de fuerza en la composición corporal y la fuerza máxima de jóvenes entrenados. Dicho estudio mostró una reducción del $9,6 \%$ en la grasa corporal, una mejora en la fuerza máxima del 3 al 31\% según el componente muscular en grupos y un aumento del 1,2\% en la resistencia muscular local, concluyendo que el entrenamiento correspondiente al circuito intermitente puede ser un método alternante eficaz para mejorar la composición del cuerpo del deportista a través del ejercicio de resistencia, y se requieren aun más estudios con muestras de mayor envergadura y en poblaciones distintas. Aunque los resultados del estudio antes citado no sean equiparables respecto al presente se puede observar que los ejercicios de fuerza si generan beneficios en la reducción de la grasa corporal, además que se observa que hubo una mejora de composición corporal mediante el ejercicio de resistencia como se lo evidenció en los datos de este estudio. (Castrillón et al., 2009). 
Martínez, et al. (2012) analizó la composición corporal y somatotipo de nadadores adolescentes federados en un total de 17 encontrando diferencias significativas $(p<0,05)$ según el sexo para todas las variables examinadas (peso, altura, pliegues, circunferencia, diámetro y longitud) en: altura, envergadura, pliegues cutáneos (tricipital, subescapular, cresta ilíaca, ileospinal, abdominal, muslo, pierna y suma de arrugas), índice biacromiobicrestal, masa corporal ósea, muscular y grasa, endomorfismo y ectomorfismo. Dicho estudio fue medido y evaluado mediante el protocolo ISAK como se lo realizó en este estudio encontrando que ciertos indicadores como pliegues, índice masa muscular presentaron diferencias estadísticamente significativas, el grupo de estudio presento ser en el somatotipo endomorfo al 100\%; el protocolo de medida es muy completo por lo que su aplicación es de utilidad al tener indicadores cuantitativos mismos que brindan una buena cantidad de información.

La investigación llevada a cabo por Lesinski y colaboradores (2017) en su trabajo relacionado con los efectos del entrenamiento de fútbol en el aspecto antropométrico, aptitud y composición físico corporal durante una temporada de fútbol en jóvenes atletas de élite: mostró que la composición corporal (es decir, la masa corporal magra, la masa de grasa corporal) varió de acuerdo con las exigencias de los respectivos períodos de entrenamiento, lo que es indicativo de cambios biopositivos. Evidencia que fue concomitante con el referente estudio además aquello los valores de composición corporal se presentaron en mayor proporción como significativos y la reducción en el total de los indicadores de gracia y de IMC lo que demostraría lo que se menciona que la exigencia el ejercicio funcional estableció biopositivad en los jugadores de futbol y su consecuente mejora de rendimiento y resistencia.(Lesinski et al., 2017)

El entrenamiento funcional cumple con la tarea de trabajar todos los movimientos del cuerpo humano y esto basado en la fuerza funcional, integrando al cuerpo como un todo, además de la perspectiva en relación con la actividad física y el aumento progresivo de la exigencia. (Manzano \& Pescador, 2017) Este criterio es fundamental dentro de los 
ejercicios funcionales mismos que se palpo con el grupo de estudio siendo los resultados posteriores lo que logran motivar en el jugador la importancia de su desarrollo y de la práctica en post del mejoramiento de su rendimiento.

\section{CONCLUSIONES}

Se analizó la variación del componente graso y muscular, mediante un programa de intervención de entrenamiento funcional, en futbolistas profesionales, encontrando una variación significativa $(p<0,05)$ antes y después del trabajo realizado mediante los ejercicios funcionales en los componentes corporales de IMC, pliegues, Porcentaje Graso (Carter, a partir de Yuhasz) y Porcentaje Muscular (Poortmans).

Además, se pudo constatar el trabajo realizado y la variación tanto en pliegues como en perímetros, en los cuales se observó también cambios significativos $(p<0,05)$ en bíceps, cresta ilíaca, abdominal, muslo, brazo flexionado y contraído, muslo medio, muslo corregido, pierna.

\section{REFERENCIAS CONSULTADAS}

Acosta, D. A., \& García, O. (2013). Kineanthropometry Applied To High Perfomance. Cub. Med. Dep. \& Cul. Fís., 8(3).

Antunes, B. S., Bianco, R., \& Lima, W. P. (2020). Treinamento funcional: Conceitos e benefícios. Revista Científica Multidisciplinar Núcleo Do Conhecimento, 69-80. https://doi.org/10.32749/nucleodoconhecimento.com.br/educacaofisica/treinamento-funcional

Cárdenas-Fernández, V. (2015). Estudio transversal evolutivo de la composición corporal en futbolistas de base y su relación con los hábitos nutricionales. [Cross-sectional evolutionary study of body composition in grassroots soccer players and its relationship with nutritional habits]. https://n9.cl/5lxer 
Revista Arbitrada Interdisciplinaria KOINONIA

Año VI. Vol VI. N². Edición Especial: Educación III. 2021

Hecho el depósito de Ley: FA2016000010

ISSN: 2542-3088

FUNDACIÓN KOINONIA (F.K). Santa Ana de Coro. Venezuela.

Cristian Alejandro Naranjo-Sánchez; Raúl Fernando Moscoso-García; Antonio Ricardo Rodríguez-Vargas

Castrillón, F. J. O., Torres-Luque, G., \& de León, F. P. (2009). Efectos de un programa de entrenamiento de fuerza sobre la composición corporal y la fuerza máxima en jóvenes entrenados. Apunts Medicina de l'Esport, 44(164), 156-162. https://doi.org/10.1016/S1886-6581(09)70126-4

Erazo Álvarez, J. C. (2021). Capital intelectual y gestión de innovación: Pequeñas y medianas empresas de cuero y calzado en Tungurahua-Ecuador. Revista De Ciencias Sociales, 27, 230-245. Recuperado a partir de https://www.produccioncientificaluz.org/index.php/rcs/article/view/37004

Erazo, J. y Narváez, C. Medición y gestión del capital intelectual en la industria del cuero - calzado en Ecuador. Revista Arbitrada Interdisciplinaria Koinonía, 5 (9), 437-467. Recuperado a partir de https://n9.cl/aeOhc

Fessi, M., Nidhal, Z., Filetti, C., Rebai, H., Elloumi, M., \& Moalla, W. (2016). Physical and anthropometric changes during pre and in-season in professional soccer players. The Journal of Sports Medicine and Physical Fitness, 56, 1163-1170.

Hammami, M. A., Abderrahmane, A. Ben, Nebigh, A., Le, E., Ounis, O. Ben, Tabka, Z., \& Zouhal, H. (2013). Effects of a soccer season on anthropometric characteristics and physical fitness in elite young soccer players. Journal of Sports Sciences, 31(6), 589-596. https://doi.org/http://dx.doi.org/10.1080/02640414.2012.746721

Leão, C., Camões, M., Clemente, F. M., Nikolaidis, P. T., Lima, R., Bezerra, P., Rosemann, T., \& Knechtle, B. (2019). Anthropometric profile of soccer players as a determinant of position specificity and methodological issues of body composition estimation. International Journal of Environmental Research and Public Health, 16(13), 1-10. https://doi.org/10.3390/ijerph16132386

Lesinski, M., Prieske, O., Helm, N., \& Granacher, U. (2017). Effects of soccer training on anthropometry, body composition, and physical fitness during a soccer season in female elite young athletes: A prospective cohort study. Frontiers in Physiology, 8(DEC), 1-13. https://doi.org/10.3389/fphys.2017.01093

Manzano, D., \& Pescador, C. (2017). Promoción de la actividad física para la salud a través del entrenamiento funcional en escolares adolescentes [Promotion of physical activity for health through functional training in adolescent schoolchildren].

Martínez-Sanz, J. M., Mielgo-Ayuso, J., \& Urdampilleta, A. (2012). Composición corporal y somatotipo de nadadores adolescentes federados [Body composition and 
Revista Arbitrada Interdisciplinaria KOINONIA

Año VI. Vol VI. N4. Edición Especial: Educación III. 2021

Hecho el depósito de Ley: FA2016000010

ISSN: 2542-3088

FUNDACIÓN KOINONIA (F.K). Santa Ana de Coro. Venezuela.

Cristian Alejandro Naranjo-Sánchez; Raúl Fernando Moscoso-García; Antonio Ricardo Rodríguez-Vargas

somatotype of federated adolescent swimmers.]. Revista Espanola de Nutricion Humana y Dietetica, 16(4), 130-136. https://doi.org/10.1016/S2173-1292(12)70087$\underline{X}$

Martínez, \&, \& Urdampilleta. (2012). Medición Antropométrica En El Deportista Y Ecuaciones De Estimaciones De La Masa Corporal. Medicion, 1(3), 4.

Miranda, R., Antunes, H., Pauli, J., Puggina, E., \& Da Silva, A. (2013). Effects of 10-week soccer training program on anthropometric, psychological, technical skills and specific performance parameters in youth soccer Effets d' un programme d' entraînement de football de dix semaines sur les. Science et Sports, 28(2), 81-87. https://doi.org/10.1016/j.scispo.2012.02.005

Rivas Borbón, M, \& Sánchez Alvarado, E. (2013). Fútbol. Entrenamiento actual de la condición física del futbolista. MHSalud, 10(2),1-131.

Rivera Sosa, J. . (2006). Futbolistas Profesionales Evaluation of the Somatotype and Proportionality of University Soccer Players. Journal of Medicine and Science of Physical Activity and Sport, 6(21), 16-28.

Rodríguez P., X., Castillo V., O., Tejo C., J., \& Rozowski N., J. (2014). Somatotype of high performance athletes of Santiago, Chile. Revista Chilena de Nutrición, 41(1), 29-39. https://doi.org/10.4067/S0717-75182014000100004

Slimani, M., \& Nikolaidis, P. T. (2019). Anthropometric and physiological characteristics of male soccer players according to their competitive level, playing position and age group: A systematic review. Journal of Sports Medicine and Physical Fitness, November, 141-163. https://doi.org/10.23736/S0022-4707.17.07950-6

Toala, M., \& Aguilar, E. (2018). Estudio del perfil antropométrico a deportistas de fútbol sala para determinar la posición de juego según el somato tipo e índice de masa corporal [Study of the anthropometric profile of indoor soccer athletes to determine the playing position according t. Revista Ciencia \& Tecnologia, 18(19), 198-210. https://doi.org/https://doi.org/10.47189/rcct.v18i19.205

C2021 por los autores. Este artículo es de acceso abierto y distribuido según los términos y condiciones de la licencia Creative Commons Atribución-NoComercial-Compartirlgual 4.0 Internacional (CC BY-NC-SA 4.0) (https://creativecommons.org/licenses/by-nc-sa/4.0/). 